\title{
Mars Dust Counter on Board ISAS PLANET-B
}

Eduard Igenbergs

LRT, TU-München, 80333 München, Germany

Sho Sasaki

Geological Institute, University of Tokyo, Hongo, Tokyo 113, Japan

Georg Färber, Franz Fischer, and Ralf Münzenmayer

$L P R$ and LRT, TU-München, 80333 München, Germany

Akira Fujiwara and Heinrich Iglseder ${ }^{1}$

ISAS, 3-1-1 Yoshinodai, Sagamihara 229, Japan

Albrecht Glasmachers

Ruhr Universität Bochum, 44780 Bochum, Germany

Eberhard Grün

MPI Kernphysik, Saupfercheckweg 1, 69117 Heidelberg, Germany

Tadashi Mukai

Kobe University, 1-1 Rokkoudai, Nada-ku, Kobe 657, Japan

Ken-ichi Nogami

Dokkyo Medical University, Mibu, Tochigi 312-02, Japan

Hideo Ohashi

Tokyo University of Fishery, 4-5-7 Konan, Minato-ku, Tokyo 108, Japan

Gerhard Schwehm and Håkan Svedhem

ESA-ESTEC, PO Box 299, 2200 AG Noordwijk, The Netherlands

Kazuo Yamakoshi ${ }^{2}$

ICRR, University of Tokyo, Midori-cho, Tanashi 188, Japan

Abstract. PLANET-B is an ISAS spacecraft which will investigate the upper atmosphere and the surroundings of Mars between 1999 and 2001 To clarify the presence and characteristics of the Martian dust ring/torus, an impact ionization dust detector will be on board PLANET-B. The detector (PLANET B Mars Dust Counter), which is an improved version of the Munich Dust Counters of HITEN and BREMSAT, will weigh only $630 \mathrm{~g}$ with an aperture area $140 \mathrm{~cm}^{2}$. The detectable mass range will be between $10^{-16} \mathrm{~g}$ and more than $10^{-6} \mathrm{~g}$ and the velocity range will be from $1 \mathrm{~km} / \mathrm{s}$ to more than $70 \mathrm{~km} / \mathrm{s}$. Since PLANET-B executes retrograde elliptic orbits close to the zodiacal plane, our detector can investigate the spatial distribution of prograde

\footnotetext{
${ }^{1}$ ZARM, Universität Bremen

${ }^{2}$ Deceased
} 




Figure 1. Expected ranges of mass and velocity of detectable dust particles by PLANET-B MDC. Capabilities of dust accelerators which will be used for calibration are also shown.

dust particles from Phobos and Deimos with relative encounter velocity as large as or higher than $1 \mathrm{~km} / \mathrm{s}$. PLANET-B MDC shall also measure the dust environment around the Earth and interplanetary and possibly interstellar dust particles.

\section{Project Overview: PLANET-B and MDC}

PLANET-B is a Japanese spacecraft which will investigate the Martian upper atmosphere and its interaction with the solar wind. A dust counter MDC (Mars Dust Counter) is planned to be on board PLANET-B (ISAS 1995). The primary purpose of $\mathrm{MDC}$ is to detect circummartian dust particles and clarify the distribution of the proposed Martian ring or torus of dust particles, which are released from Phobos/Deimos through impact of interplanetary particles and/or circummartian particles themselves (e.g., Soter 1971, Horányi et al. 1990, Juhász et al. 1993. Ishimoto \& Mukai 1994, Sasaki 1994, Hamilton 1996, Ishimoto 1996). $\mathrm{MDC}$ : will also measure interplanetary and interstellar dust particles.

PLANET-B is scheduled to be launched in August 1998. After several flybys with the moon, it will leave the Earth on December 20, 1998. It will arrive at Mars on October 11, 1999, and then it will execute scientific measurements around Mars for at least two years (ISAS 1995).

During the first 4 -month parking phase around the Earth, MDC will measure interplanetary, interstellar and circumterrestrial particles; the results can be compared with previous data, especially those obtained by MDC-HITEN

Throughout the 10-month transfer orbit, MDC will measure both interplanetary and interstellar dust particles. Note that the Cassini spacecraft will pass through the interplanetary space between the Earth and Mars at the same time. If CDA (Cassini Dust Analyzer) will be operated during the cruising phase, MDC data can be compared with CDA data. 
Around Mars, PLANET-B will execute elliptic retrograde orbits whose periapsis is $130-150 \mathrm{~km}$ above the Martian surface and apoapsis is $15 R_{M}\left(R_{M}\right.$. Martian radius). The orbits, which are close to the zodiacal plane, will intersect with the orbits of Phobos and Deimos twice respectively during the first two years of operation. The detection zone with large periapsis can fully cover the expected distribution of dust particles from Phobos or Deimos. Moreover, relative velocity between retrograde PLANET-B and prograde ring dust can be larger than $1 \mathrm{~km} / \mathrm{s}$, which is suitable for impact ionization detection. It would be difficult to detect ring particles by a dust detector on a spacecraft executing a prograde circular orbit around Mars because of the small relative velocity

\section{Instrument}

The proposed instrument is an improved version of the Munich Dust Counter flown on the Japanese MUSES-A-satellite (HITEN) and on the German BREMSAT (Igenbergs et al. 1991). MDC PLANET-B (Mars Dust Counter) is a multicoincidence impact-ionization detector measuring particles with mass between $10^{-16}$ and more than $10^{-6} \mathrm{~g}$ and velocity between less than 1 and more than $70 \mathrm{~km} / \mathrm{s}$ (Fig. 1). These mass and velocity ranges cover the expected mass and impact velocity of circumplanetary, interplanetary, and interstellar dust particles. HITEN experiments detected not only interplanetary and circumterrestrial particles but also interstellar particles (Svedhem et al. 1996). Our improved detector will obtain reliable, quantitative data on dust particles, especially those orbiting around Mars.

MDC PLANET-B consists of an electronic box and a sensor box (Fig. 2). The size of MDC is $135 \times 126 \times 177 \mathrm{~mm}^{3}$ with an aperture of $125 \times 116 \mathrm{~mm}^{2}$ which is covered with a steel grid. The weight and power consumption of MDC are $630 \mathrm{~g}$ and $3.5 \mathrm{~W}$, respectively. The MDC will be mounted with four flanges onto the side panel of the spin-stabilized spacecraft (Fig. 2). The aperture direction of the MDC sensor is $135^{\circ}$ from the spacecraft spin axis, around which the sensor will scan within its field of view. Since the spacecraft spin axis will look at the sun (at circumterrestrial orbits) or at the Earth (at transfer and circummartian orbits), the sensor box is always free of solar illumination. This can effectively eliminate noise signals due to photoionization.

The MDC experiment measures electrical charges of plasma generated by high velocity impacts of particles on a gold-coated surface. The MDC sensor consists of a target plate and two charge collector plates which are biased by negative and positive voltages $( \pm 200 \mathrm{~V})$. The impact plasma is separated into positive ions detected by the negative collector and electrons and negative ions by the positive collector Mass and velocity of the particle are estimated from the total charges (or pulse height) and rise times of positive and negative charges.

\section{Discussions - Current Prediction}

Recent theoretical studies show that solar radiation pressure together with Martian oblateness should enhance the amplitude of orbital eccentricity of ring particles. At Phobos' orbit, particles smaller than $20 \mu \mathrm{m}$ are quickly trapped by Mars. If the dust source should be solely the secondary ejecta from interplanetary dust 

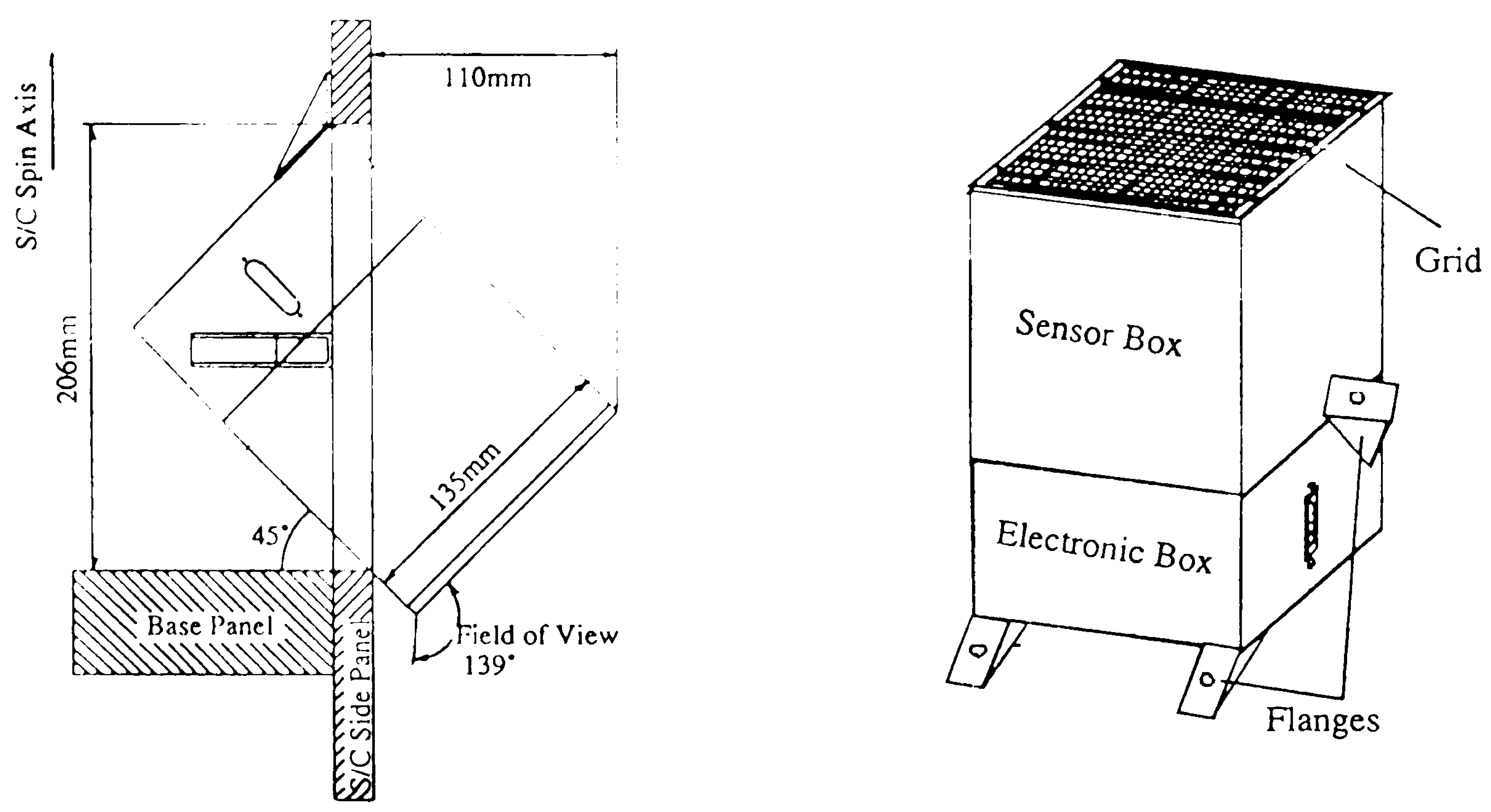

Figure 2. Configuration of $\mathrm{MDC}$ and its attachment to the PLANET-B spacecraft

collisions onto satellites, the abundance of circummartian particles would be small and the detected number of them by MDC should be much smaller than that of interplanetary particles. However, collisions of ring particles onto Phobos and Deimos may produce new dust particles because of satellite low escape velocity, and the dust ring/torus is self-sustained (Sasaki 1994 \& 1996, Hamilton 1996, Ishimoto 1996). If this mechanism is efficient, dust abundance will be high enough that MDC can detect particles at each crossing of the ring/torus.

Orbital calculations suggest that dust particles from Phobos would form a thin ring (inclination $i \sim 0.01$ ) containing particles with dimensions $20-100 \mu \mathrm{m}$, whereas particles from Deimos would form a thick torus $(i \sim 0.2)$ containing even smaller particles. Determination of particle mass and velocity by MDC PLANET-B will not only confirm circummartian particles but also distinguish particles from Phobos and those from Deimos.

\section{References}

Hamilton, D. P. 1996, Icarus in press and in this volume

Horányi, M.et al. 1990, Geophys. Res. Lett., 17, 853

Igenbergs E. et al. 1991, In Origin \& Evolution of Interplantary Dust, 15 \& 45 ISAS 1995, PLANET-B INTERIM REPORT

Ishimoto. H. and Mukai, T. 1994, Planet. Space. Sci., 42, 691

Ishimoto. H. 1996, Icarus in press and in this volume

Juhász, A, et al. 1993, J Geophys. Res., 98, 1205

Sasaki. S. 1994, Proc. 27th ISAS Lunar Planet. Symp., 47

Sasaki, S. et al. 1996, in this volume

Soter, S. 1971, Cornell Center Radiophys. \& Space Res. Rep. 462

Svedhem, H. et al. 1996, in this volume 